\title{
The EUSO-Balloon instrument
}

\author{
Valentina Scotti* \\ Università degli Studi di Napoli Federico II, Italy \\ INFN, sezione di Napoli, Italy \\ E-mail: scottivena.infn.it
}

\section{Giuseppe Osteria}

INFN, sezione di Napoli, Italy

E-mail: osteria@na.infn.it

\section{for the JEM-EUSO Collaboration}

EUSO-Balloon is a pathfinder mission for JEM-EUSO (Extreme Universe Space Observatory on-board the Japanese Experiment Module), the near-UV telescope proposed to be installed on board the International Space Station (ISS) before the end of this decade. The main objective of this pathfinder mission is to perform a full scale end-to-end test of all the key technologies and instrumentation of JEM-EUSO detectors and to prove the entire detection chain.

The JEM-EUSO instrument consists of an UV telescope designed to focus the signal of the UV tracks generated by Extreme Energy Cosmic Rays propagating in Earth's atmosphere, onto a finely pixelized UV camera. The EUSO-Balloon instrument, smaller than the one designed for the ISS, is currently developed as a payload of a stratospheric balloon operated by the French Centre National d'Études Spatiales (CNES) and will be launched during the CNES flight campaign in August 2014. This telescope will point towards the nadir from a float altitude of about $40 \mathrm{~km}$. With its Fresnel Optics and Photo-Detector Module, EUSO-Balloon will monitor a $12^{\circ} \times 12^{\circ}$ wide field of view in a wavelength range between 290 and $430 \mathrm{~nm}$, at a rate of 400'000 frames/sec.

In this paper, we will review the main stages of the signal processing of the EUSO-Balloon instrument: the photodetection, the analog electronics, the trigger stages, which select events while rejecting random background, the electronic acquisition system which performs the data management and the monitoring, allowing the instrument control during operation.

Technology and Instrumentation in Particle Physics 2014,

2-6 June, 2014

Amsterdam, the Netherlands

\footnotetext{
*Speaker.
} 


\section{The JEM-EUSO project}

The Extreme Universe Space Observatory (EUSO) on-board the International Space Station (ISS) Japanese Experiment Module is a new type of observatory which aims to observe fluorescence and Cherenkov photons generated by air showers created by Extreme Energy Cosmic Rays, EECRs $\left(E>5 \times 10^{19} \mathrm{eV}\right)$, in the Earth's atmosphere. The main objective of JEM-EUSO is to investigate the nature and origin of EECRs: to identify their sources by arrival direction analysis and to measure their energy spectra with a high collecting power, beyond any other previous or planned experiment so far.

JEM-EUSO is designed to monitor from space, looking towards nadir during night-time, the Earth's atmosphere to detect the UV (290-430 nm) tracks generated by the Extensive Air Showers (EAS) propagating through the atmosphere. By imaging, with a time resolution of the order of ms, the fluorescence and Cherenkov photons of the EAS, the energy, arrival direction and nature of the primary EECR particle will be reconstructed. The instrument is planned to be attached to JEM/EF (Exposed Facility) of ISS during the first half of 2017 for a three years long mission. Placed at an altitude of about $400 \mathrm{~km}$ from the Earth's surface, JEM-EUSO will orbit the earth, with a speed of $\sim 7 \mathrm{~km} / \mathrm{s}$, every $\sim 90$ minutes and will be able to reach an instantaneous aperture of about $6-7 \times 10^{5}$ $\mathrm{km}^{2} \mathrm{sr}$, beyond the practical limit of any ground-based CRs observatory.

The JEM-EUSO instrument is a wide-angle refractive telescope in the near-UV wavelength region observing fluorescence and Cherenkov photons generated by air showers created by UHECRs in the Earth's atmosphere. It consists basically of four parts:

- Optics: two high transmittance optical Fresnel lenses (diameter of $2.35 \mathrm{~m}$ ) focusing the arriving UV photons onto the Focal Surface (FS);

- FS detector: 4936 Multi-Anode Photo-Multiplier Tubes (MAPMTs) of 64 pixels each;

- Electronics: FS electronics, trigger, data acquisition and controls;

- Mechanical Structure.

An atmosphere monitoring system and a calibration system complete the apparatus.

The mission consortium includes 17 countries and is lead by RIKEN (Japan), in coordination with the Japanese Space Agency (JAXA). Further details about JEM-EUSO mission and instrument can be found in [1].

\section{The EUSO-Balloon pathfinder}

EUSO-Balloon is developed by the JEM-EUSO consortium as a demonstrator for technologies and methods featured in the space instrument [2]. This balloon-borne instrument points toward the nadir from a float altitude of about $40 \mathrm{~km}$. With its Fresnel optics and PDM, the instrument monitors a $12 \times 12$ degree wide field of view. The EUSO-Balloon mission has been proposed by a collaboration of three French laboratories (APC, IRAP and LAL) involved in the international JEM-EUSO consortium. The instrument is being built by various institutes of the JEM-EUSO collaboration. Balloon flights will be performed by the balloon division of the French Space Agency CNES, the first flight is scheduled in 2014. 
The main objective of the EUSO-Balloon mission is to perform a full scale end-to-end test of all the key technologies and instrumentation of JEM-EUSO detectors and to prove the global detection chain. It will measure the atmospheric and terrestrial UV background components, in different observational modes, testing and adjusting trigger and switching algorithms and the acquisition capability of the IR camera.

\section{Description of the instrument}

The prototype instrument will consist of a Fresnel Optics made from three PMMA square flattype lenses, a focal plane detector made from a single PDM of 2304 pixels with front-end read-out performed by 36 ASICs while readout tasks are performed by an FPGA board that stores the data in round buffer, and a Data Processor (see Fig. 1). The performances of EUSO-Balloon will be

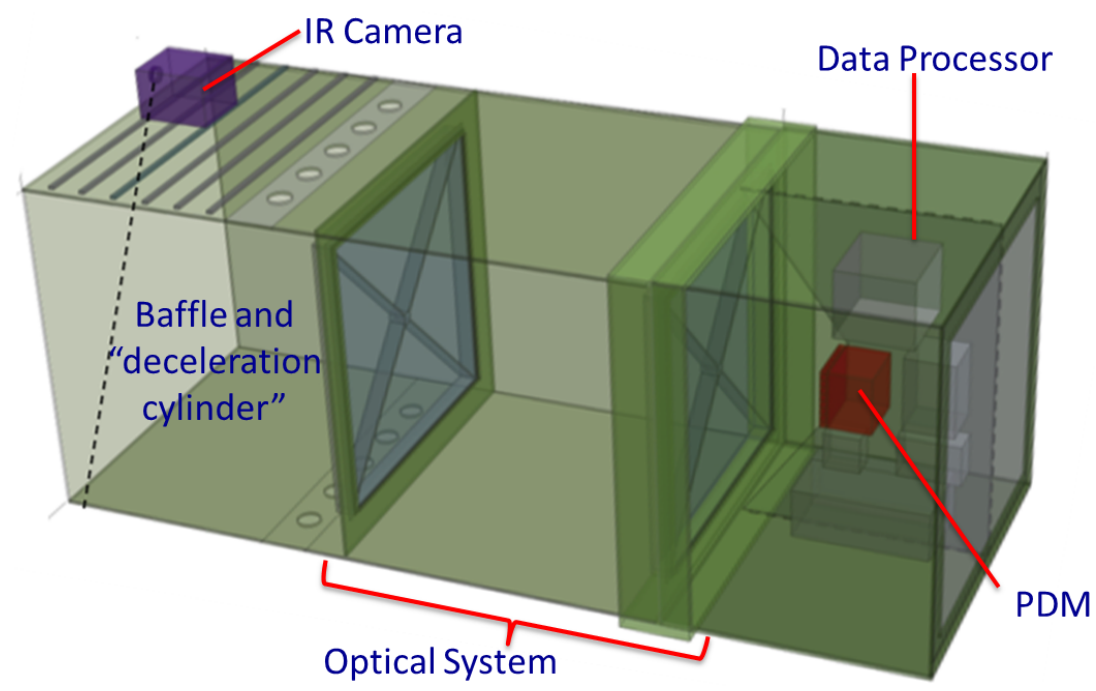

Figure 1: A schematic view of the EUSO-BALLOON telescope.

maximized keeping parameters as close as possible to JEM-EUSO, as is shown in table 1.

The instrument monitors a $12^{\circ} \times 12^{\circ}$ wide field of view in a wavelength range between 290 and $430 \mathrm{~nm}$, at a rate of 400000 frames/s. The pixel size in the field of view is $0.25^{\circ} \times 0.25^{\circ}$, corresponding to $175 \mathrm{~m} \times 175 \mathrm{~m}$ on the ground for a float level of $40 \mathrm{~km}$. In order to monitor the actual cloud covers, a co-aligned IR camera will observe the field of view of the main instrument for the EUSO-Balloon prototype.

The UV light collected by the optical system is detected by the MAPMTs of the PDM. The PDM is mounted on a translation stage inside the telescope to adjust its position respect to the lenses. Besides monitoring the UV background, the PDM detects candidate shower events by the first trigger implemented in the PDM-board. Data acquired by the PDM are transferred via the PDM-board to the DP component which performs data management and storage, instrument control and commanding. A block diagram of the instrument summarizing the subsystems, and the main sub-assembly items is shown in Figure 2. The development of all components and subassemblies is based on similar JEM-EUSO components and sub-assemblies. The three main com- 
Table 1: Comparison of the principle characteristics between JEM-EUSO and EUSO-BALLOON. The field of view of EUSO-BALLOON, and hence its pixel size, has been dimensioned to measure a background level comparable to the one expected for JEM-EUSO.

\begin{tabular}{|c|c|c|}
\hline & JEM-EUSO & EUSO-Balloon \\
\hline Number of PDMs & 14 & 31 \\
\hline Flight Altitude $[\mathrm{km}]$ & 420 & 40 \\
\hline Diameter of Optics [m] & 2.5 & 1 \\
\hline Field of View / PDM & $3.8^{\circ}$ & $12^{\circ}$ \\
\hline PDM@ ground [km] & 28.2 & 8.4 \\
\hline Field of View / pixel & $0.08^{\circ}$ & $0.25^{\circ}$ \\
\hline Pixel@ ground [km] & 0.580 & 0.175 \\
\hline Signal w/r JEM-EUSO & 1 & 17.6 \\
\hline BG w/r JEM-EUSO & 1 & $0.9-1.8$ \\
\hline S/ $\sqrt{N}$ w/r to JEM-EUSO & 1 & $20-10$ \\
\hline Threshold Energy [eV] & $3 \times 10^{19}$ & $1.5 \div 3 \times 10^{18}$ \\
\hline
\end{tabular}

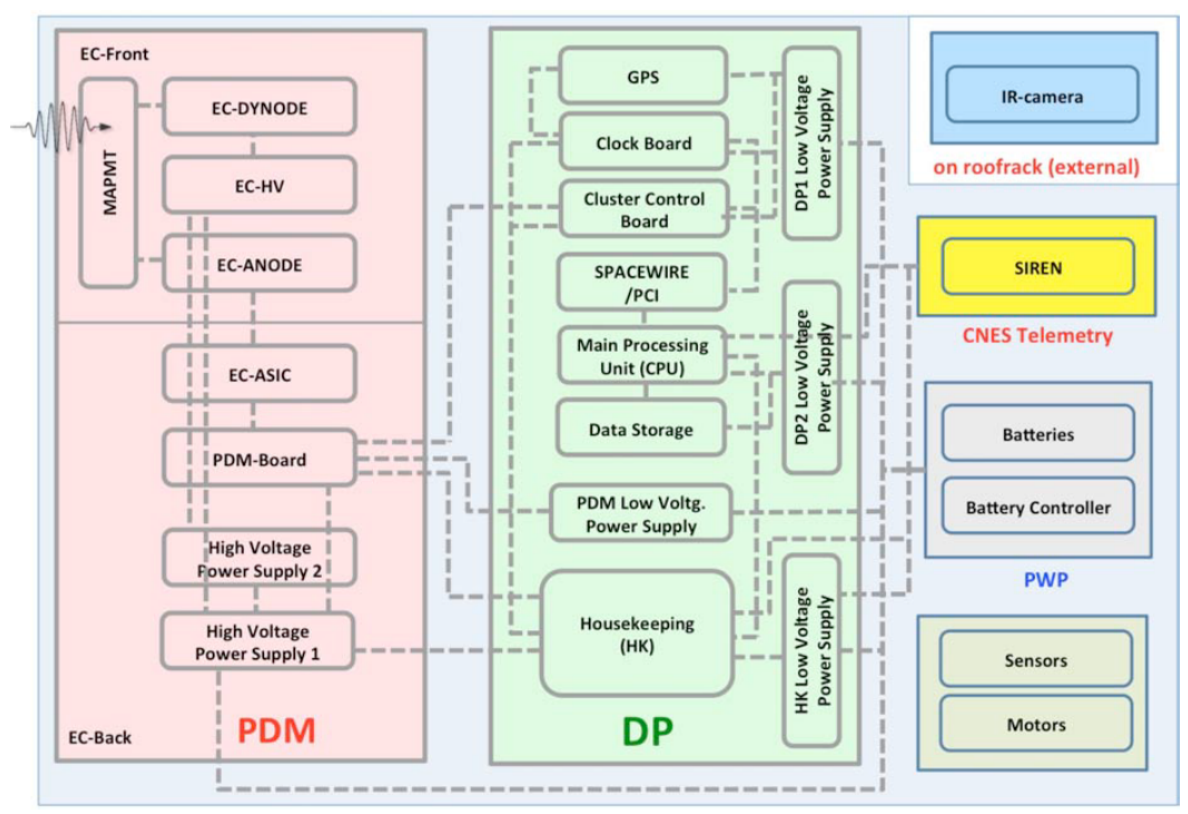

Figure 2: Functional Block Diagram of the EUSO-BALLOON Instrument.

ponents of the instrument, the Optical System, the PDM and the DP are, in turn, divided into several subsystems. In the next section we will describe the main components in more details.

Integrating one of the Fresnel lenses as a port-hole window directly into the telescope structure will make the electronics compartment (with its crucial payload elements such as the PDM) entirely water tight without moving parts and will permit offshore recovery (later, possibly long duration balloon flights).

The telescope is composed of a self-contained, watertight instrument booth containing PDM, electronics, telemetry and an optics module serving as optical bench for the lenses. The structure of 
both the instrument booth and the optics module is made of $10 \mathrm{~mm}$ "Fibrelam" aerospace panels. The total weight of structure, instrument, dedicated electronics, telemetry systems and batteries is around $250 \mathrm{~kg}$.

During the balloon flight operation, the instrument will be controlled from ground by an operator using a control program interfaced to the TC/TM system (Telecommand and Telemetry) SIREN NOSYCA of CNES.

\subsection{Optical system}

The optical system focuses the incoming UV photon toward a pixel of the detector on the optical focal surface. The distance between the front surface of the front lens and the focal surface is $1620.7 \mathrm{~mm}$. Each lens has a $1 \mathrm{~m}^{2}$ square surface, a thickness of $8 \mathrm{~mm}$ and a weigh of $9.6 \mathrm{~kg}$.

\subsection{Photo Detector Module}

The PDM (Fig.3) is composed of 36 MAPMTs containing 64 anodes each and their associated electronic chain [4]. On the mechanical structure the MAPMTs are grouped in 9 Elementary Cells (EC) which supply the 14 voltages needed for each of them and collect signals from their anodes. These signals are transmitted to the SPACIROC ASIC for processing [3]. The output signals of all the ASICs are delivered to the PDM board. The PDM is equipped with two High Voltage Power Supply (based on a Cockroft-Walton design) providing the necessary voltages to MAPMT dynodes.

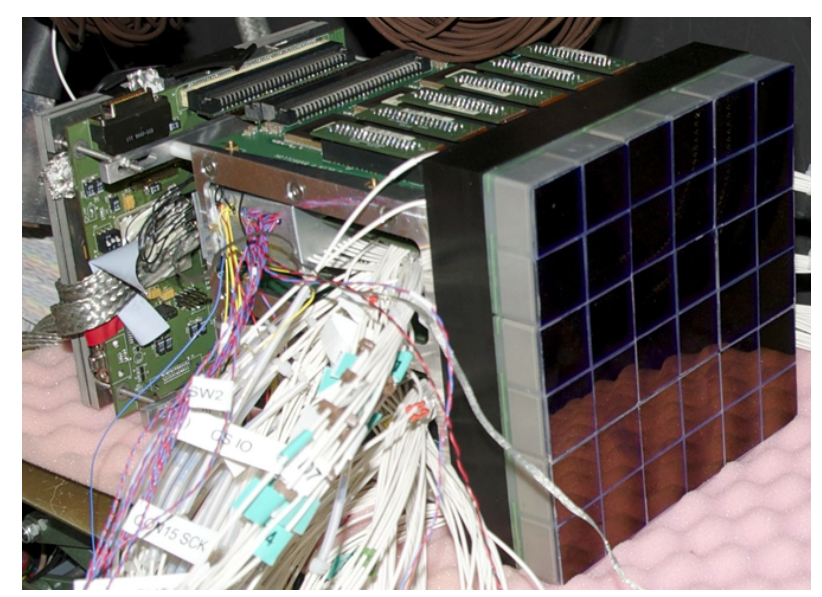

Figure 3: The whole PDM of the EUSO-Balloon instrument.

The MAPMTs sense the UV photons arriving through the lenses. An optical filter "SCHOTT BG3", which transmits the UV photon and absorbs the visible light, is glued on each MAPMT. The MAPMTs are manufactured by Hamamatsu and have been developed specifically for the JEMEUSO mission. Their sensitivity is as low as a few tenths of photon and their dynamic range can extend up to few thousands photons per $\mu$ s when working at their nominal high gain of $10^{6}$.

The ASIC has been designed by Omega group at LAL in AMS $0.35 \mu \mathrm{m}$ SiGe technology [3]. It reads out MAPMTs anodes signals. It has two main purposes: counting the number of single photon events reaching each pixel of the MAPMTs and measuring the intensity of photon flux by 
performing charge to time conversion. The ASIC digitizes the analog signal inputs and sends the data to the PDM board via dedicated digital connections.

The PDM board handles all the EC units. It performs the $1^{\text {st }}$ level trigger looking for pixels firing for several contiguous Gate Time Units(GTU) on the PDM. It manages the interfaces with the EC boards, Control Cluster Board (CCB) as well as the HV system.

\subsection{Data Processor}

The DP system is the component of the electronics subsystem which includes most of the digital electronics of the instrument. It controls the front-end electronics, performs the $2^{\text {nd }}$ level trigger filtering, tags events with arrival time and payload position, manages the data storage, measures live and dead time of the instrument, provides signals for time synchronization of the event, performs housekeeping monitoring and handles the interfaces to the TC/TM system. The DP system has to operate at high altitude in unpressurised environment and this represents a technological challenge for heat dissipation. To cope with thermal problems, we use a passive cooling system that exchanges heat directly from the modules through the mechanics of the DP box to the gondola structure.

The DP functionalities are obtained by connecting different specialized items, which form a complex system. The main sub-assembly items are CCB [7], CPU and Data Storage[6], Data storage, House-Keeping system, Clock Board [8], GPS receiver and Data Processor Power Supply (DP-LVPS). A block diagram of the DP is shown in Fig. 4. Further details about DP subsystems and working mode can be found in [5]

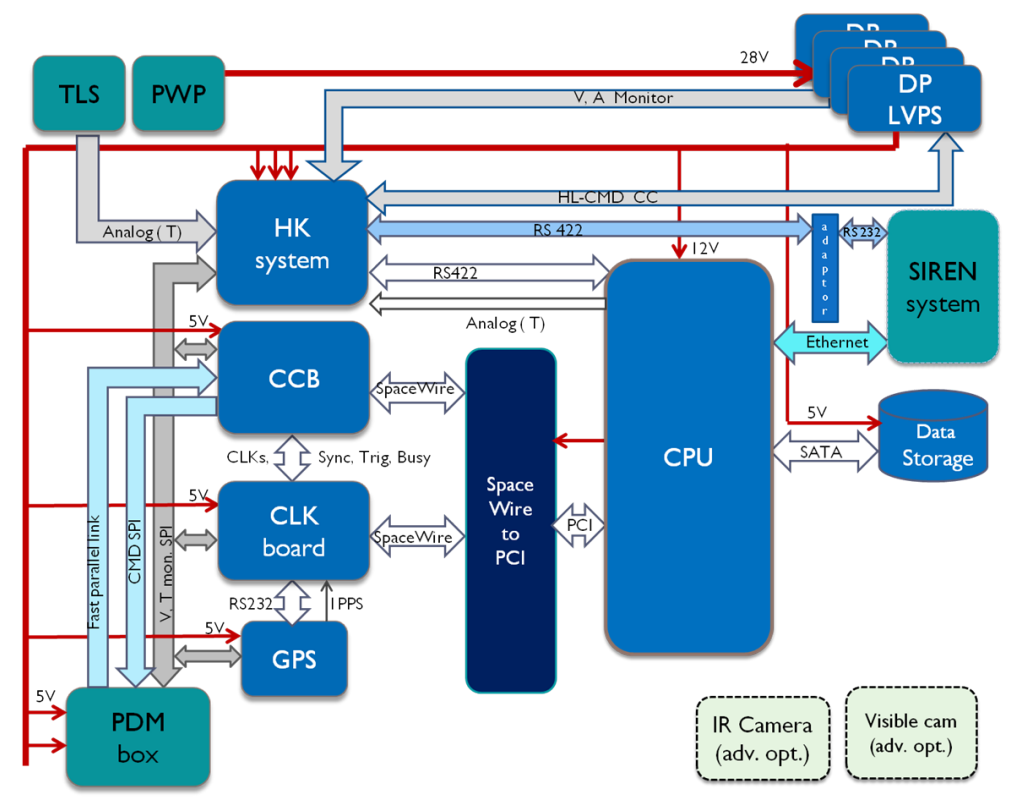

Figure 4: Functional block diagram of the Data Processor.

\section{Instrument integration and tests}

The flight model of all the sub-assemblies of the instrument and their spares have been manu- 
factured in different laboratories of most of the countries composing the JEM-EUSO collaboration. Each sub-assembly has been tested by using hardware emulators as external interface. LVPS have been tested by simulating the maximum load and flight models of the cables have been produced and tested. Finally, environmental test have been performed for all the sub-assemblies as stand alone systems.

After fabrication, the the optics and the MAPMTs were calibrated with great accuracy. The key goal of the calibration is to relate a measured digitised signal into the true number of photons impinging on the focal surface or on the first lens.

The flight model of the DP has been integrated and tested in Naples in 2013. All the mechanical interfaces, the electrical interfaces as well as the cables have been successfully tested. The DP has been delivered to APC (Paris) in October 2013 to integrate it with the PDM.

The interface between PDM and DP is complex and it includes several connections. The PDM-Board-CCB interface is the key one since it allows the communication between PDM and DP systems. Commands are sent from CCB to PDM through a serial SPI link. The PDM, on request, send data to CCB through a parallel bus. This interface was continuously tested while the firmware of the PDM board was developed or adapted. It was checked that the configuration of the ASICs was properly performed, the data request commands were properly served and information sent were meaningful and the data transfer of thousands of events could be done successfully. These tests not only involved the CCB and the PDM board but also the rest of the DP chain and the EC-ASIC boards. The first successful test of the whole electronics chain (PDM + DP) took place in March 2014 at APC. It ended a long period of tests and integration of the different elements of the 2 sub-systems. The complete PDM was in the black box connected to the complete DP via a feedthrough. Meaningful measurements were taken with and without light. End of April and beginning of May, functional tests and preliminary performance tests were performed at APC before shipment to Toulouse.

\subsection{Full integration tests}

In May 2014, the whole system was integrated at IRAP, in Toulouse, assembling optics, PDM, DP and Power Pack. The aim of the integration was to perfom end-to-end tests, we managed to:

- operate the instrument from flight batteries with flight-plug;

- measure the width of the Point Spread Function (PSF) as a function of the distance between the second lens and PDM for several wavelengths in order to find the best focal spot and to align optics and PDM;

- map the PSF at different wavelengths and incident angles;

- measure the overall (end-to-end) efficiency for $375 \mathrm{~nm}$;

- test the system with pulses, external triggers (GPS), and pulse-intensities to match the dynamic range;

- test all the interfaces with the TC/TM system: we successfully simulated the "in flight" operations successfully performed and the TM data were correctly received. 

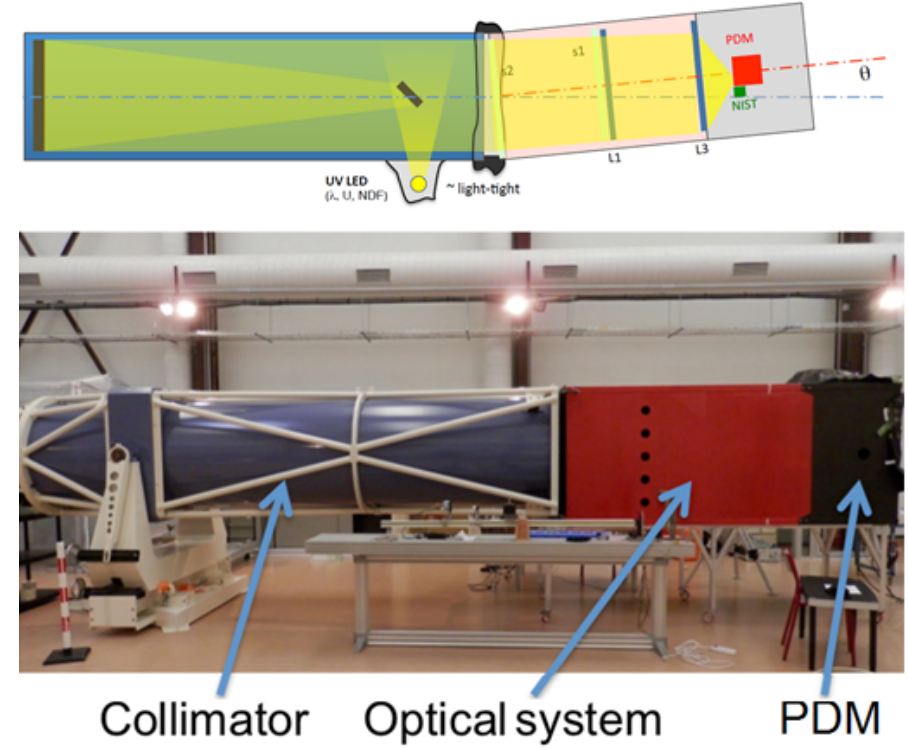

Figure 5: The whole instrument assembled for end-to-end test at IRAP (Toulouse).

\section{Status and plans}

The whole EUSO-Balloon Instrument has been assembled and tested. It will measure the spatial structure of the background at the scale of the footprint of the pixel of JEM-EUSO. Everything is on schedule for the first launch from Timmins in August 2014.

\section{References}

[1] The JEM-EUSO Collaboration, The JEM-EUSO Mission, in proceedings of $33^{\text {nd }}$ International Cosmic Rays Conference, 2013.

[2] G. Osteria and V. Scotti for the JEM-EUSO Collaboration, Euso Balloon: a pathfinder mission for the JEM-EUSO experiment, NIM A 732 (2013) 320, DOI: 10.1016/ j.nima.2013.05.124.

[3] S. Blin, SPACIROC3: A Front-End Readout ASIC for JEM-EUSO cosmic ray observatory, in proceedings of Technology and Instrumentation in Particle Physics 2014.

[4] H. Miyamoto, Performance of the EUSO-BALLOON Front-End Electronics, in proceedings of Technology and Instrumentation in Particle Physics 2014.

[5] V. Scotti and G. Osteria for the JEM-EUSO Collaboration, The data processor of the EUSO-Balloon experiment, JINST 9 (C03034) [doi : $10.1088 / 1748-0221 / 9 / 03 / C 03034]$.

[6] V. Scotti and G. Osteria for the JEM-EUSO Collaboration, The Data Processor of the JEM-EUSO pathfinders, in proceedings of International Conference on Advanced Technology and Particle Physics, 2013.

[7] J. Bayer for the JEM-EUSO Collaboration, Second level trigger and Cluster Control Board for the JEM-EUSO mission, in proceedings of $33^{\text {nd }}$ International Cosmic Rays Conference, 2013.

[8] V. Scotti and G. Osteria for the JEM-EUSO Collaboration, The JEM-EUSO time synchronization system, Nuclear Instruments and Methods in Physics Research A 718 (2013) 248. 